\title{
Fuzzy Collaborative Clustering-Based Ranking Approach for Complex Objects
}

\author{
Shihu Liu, ${ }^{1}$ Xiaozhou Chen, ${ }^{2}$ Tauqir Ahmed Moughal, ${ }^{3,4}$ and Fusheng Yu ${ }^{4}$ \\ ${ }^{1}$ School of Mathematics and Computer Science, Yunnan Minzu University, Kunming 650500, China \\ ${ }^{2}$ Key Laboratory of IOT Application Technology of Universities in Yunnan Province, Yunnan Minzu University, \\ Kunming 650031, China \\ ${ }^{3}$ Department of Mathematics and Statistics, Allama Iqbal Open University, Islamabad 44000, Pakistan \\ ${ }^{4}$ School of Mathematical Sciences, Beijing Normal University, Beijing 100875, China \\ Correspondence should be addressed to Shihu Liu; liush02@126.com
}

Received 14 March 2015; Revised 4 July 2015; Accepted 6 July 2015

Academic Editor: Laura Gardini

Copyright ( 2015 Shihu Liu et al. This is an open access article distributed under the Creative Commons Attribution License, which permits unrestricted use, distribution, and reproduction in any medium, provided the original work is properly cited.

\begin{abstract}
This paper makes a discussion on the ranking problem of complex objects where each object is composed of some patterns described by individual attribute information as well as the relational information between patterns. This paper presents a fuzzy collaborative clustering-based ranking approach for this kind of ranking problem. In this approach, a referential object is employed to guide the ranking process. To achieve the final ranking result, fuzzy collaborative clustering is carried on the patterns in the referential object by using the collaborative information obtained from each ranked object. Since the collaborative information of ranking objects is represented by cluster centers and/or partition matrices, we give two forms of the proposed approach. With the aid of fuzzy collaborative clustering, the ranking results can be obtained by comparing the difference of the referential object before and after collaboration with respect to ranking objects. One can find that this proposed ranking approach is totally different from the previous ranking methods because of its completely collaborative clustering mechanism. Moreover, some synthetic examples show that our proposed ranking algorithm is valid.
\end{abstract}

\section{Introduction}

For decision makers, the goal of ranking is to discover a mechanism that induces an increasing or decreasing order over the data set of given objects to be ranked. Ranking problem appears in many fields such as information retrieval [1], selection and evaluation [2,3], image similarity measure [4], and income distribution [5]. It can be easily found that, during the ranking process, only the attribute information is usually considered. Thereinto, the attribute information may be given by fuzzy numbers [6,7], intuitionistic fuzzy numbers [8], or interval-valued intuitionistic fuzzy numbers [9]. Generally speaking, in these cases, the ranking problem can be regarded as a multiple criteria decision making problem $[10,11]$, and the detailed decision making process can be executed by many approaches [11-13].
Most ranking algorithms receive attribute information and output a real-valued sequence as the ranking results. Sometimes, besides the attribute information, the relational information of the patterns is also provided for the ranking, especially in the fields of machine learning [14-16]. Up to now, there have been several developments in theory and algorithms for learning over relational information. For example, Agarwal [17] developed an algorithmic framework for learning ranking functions on graph data; Mihalcea [18] presented an innovative unsupervised method for automatic sentence extraction using graph-based ranking algorithms; Lee et al. [19] focused on the flexibility of recommendation and proposed a graph-based multidimensional recommendation method; Agarwal [20] considered the graph learning problems through ranking objects relative to one another, and so on $[21,22]$. No matter what problem to investigate or which 
algorithm to construct, in general, the patterns that need to be sorted are described by only one kind of information: attribute information or relational information.

Obviously, the above-mentioned methods are concentrated on attribute information or relational information separately. In practical terms, it is not enough when the attribute information and relational information of patterns are available at the same time. In fact, there have been many ranking problems that need to take both of these types of information into consideration, for the purpose of improving knowledge recognition degree.

Bearing this in mind, in this paper, we make a discussion on the ranking problem of complex objects. Each complex object consists of some simple patterns which are described by attribute information as well as the relational information between patterns. Because of the complexity of the objects, inspired by the famous TOPSIS method [23], in our proposed ranking approach, a referential object is proposed in advance. Hereinto, the number of patterns of the referential object is the same as that of each complex object. To fully fulfill the ranking problem, the fuzzy collaborative clustering mechanism is proposed and it is carried on the patterns in the referential object by using the collaborative information. The collaborative information refers to the partition matrices and/or cluster centers with respect to the complex objects, and it can be employed freely during the ranking process. During the process of ranking, the essence of the ranking algorithm is to compare the difference of the referential object before and after collaboration with respect to the ranking objects. Throughout the ranking process, only the collaborative information participates in the sequence calculation.

The remainder of this paper is organized as follows. In Section 2, we recall some basic concepts, such as the mathematical description of complex object and fuzzy collaborative clustering. In Section 3, we discuss the ranking problem about complex objects when the collaborative information is cluster centers that derived from the corresponding complex object by some clustering approaches. In Section 4, the partition matrices are regarded as the collaborative information and corresponding ranking approach is constructed. In Section 5, some synthetic examples are simulated to illustrate the validity of the proposed ranking approaches. Finally, Section 6 concludes this paper.

\section{Preliminaries}

In this section, at first we introduce the concept of complex objects and then make a brief review of the fuzzy collaborative clustering algorithm. For simplicity, here we ignore the presentation of fuzzy $c$-means clustering algorithm [24-26], not to mention other associated research topics [27-29].

2.1. Mathematical Description of Complex Objects. Mathematically, the complex object can be expressed as a tuple $G=$ $(X, E)$, where $X=\left\{x_{1}, x_{2}, \ldots, x_{n}\right\}$ is a nonempty finite set and $x_{i}$ for $i=1,2, \ldots, n$ are patterns, $E=\left\{e_{i j} \mid i, j=1,2, \ldots, n\right\}$ is a relational information set, and $e_{i j} \in E$ for $i, j=1,2, \ldots, n$ is the possible relational information between the patterns $x_{i}$ and $x_{j}$.
Formally, if $x_{i} \in X$ is nothing but a symbolic representation of the $i$ th pattern, then the relational information set $E$ can be only used during the process of data analysis. If not, the pattern $x_{i} \in X$ can be expressed as $x_{i}=\left(x_{i 1}, x_{i 2}, \ldots, x_{i m}\right)$, where $x_{i j}$ represents the value of $x_{i}$ with respect to the attribution $a_{j}$. In general, the value $x_{i j}$ may not be a real number. Sometimes it may be a set [30], an interval [31], or a fuzzy number $[32,33]$, and so forth.

Certainly, the foregoing proposed data representation formats are also suitable for the entries in relational information set $E$. Particularly, the relationship between any two patterns may be described by more than one relation; that is, $E=$ $\left\{E_{1}, E_{2}, \ldots, E_{k}\right\}$ with $k \geq 1$, where $E_{i} \in E$ describes the $i$ th relationship between patterns. Notice that, in this paper, we adhere to the hypothesis that $x_{i j}$ for $i=1,2, \ldots, n$ and $j=$ $1,2, \ldots, m$ is a real number, $e_{i j}$ for $i, j=1,2, \ldots, n$ belongs to unit interval $[0,1]$, and $k=1$ for complex object $G=(X, E)$.

2.2. Fuzzy Collaborative Clustering. It is well known that the nature of clustering analysis is to find out the potential structure of the data. If one wants to search for the common structure of some different data sets while the information of these data sets cannot be shared freely, the collaborative clustering algorithm $[34,35]$ plays an important role.

One has that the collaborative clustering can be divided into horizontal collaborative clustering, vertical collaborative clustering, and hybrid collaborative clustering. Because we have assumed that the number of patterns for each ranking object is equal, in what follows, we will use the horizontal collaborative clustering algorithm; thus we give a brief introduction of it.

Given that $X[1], X[2], \ldots, X[p]$ are $p$ data sets, and there are $n$ patterns in each $X[i i]$ for $i i=1,2, \ldots, p$, the collaborative mechanism based objective function can be constructed as

$$
\begin{aligned}
& Q[i i] \\
& \qquad=\sum_{i=1}^{c} \sum_{k=1}^{n} u_{i k}^{2}[i i] d_{i k}^{2}[i i] \\
& \\
& \quad+\sum_{\substack{j j=1 \\
j j \neq i i}}^{p} \alpha[i i, j j] \sum_{i=1}^{c} \sum_{k=1}^{n}\left(u_{i k}[i i]-u_{i k}[j j]\right)^{2} d_{i k}^{2}[i i],
\end{aligned}
$$

where $d_{i k}[i i]=\left\|x_{k}[i i]-v_{i}[i i]\right\|$ is the distance between the pattern $x_{k}[i i]$ and the cluster center $v_{i}[i i]$ in $X[i i]$ and the nonnegative parameter $\alpha[i i, j j]$ represents the collaborative degree of $X[i i]$ to $X[j j]$.

The concrete optimization process of objective function in (1) is the same as that of fuzzy $c$-means clustering algorithm [24-26] or partial supervised fuzzy clustering algorithm [36, 37]: applying Lagrange multiplier method. Just as [35] did, the partition matrix $U[i i]$ can be expressed as

$$
\begin{aligned}
u_{i k}[i i]= & \frac{\varphi_{i k}[i i]}{1+\phi[i i]} \\
& +\frac{1-(1 /(1+\phi[i i])) \sum_{j=1}^{c} \varphi_{j k}[i i]}{\sum_{j=1}^{c}\left(d_{i k}^{2}[i i] / d_{j k}^{2}[i i]\right)},
\end{aligned}
$$


where $\varphi_{i k}[i i]=\sum_{i i \neq j j} \alpha[i i, j j] u_{i k}[i i]$ and $\phi[i i]=\sum_{i i \neq j j} \alpha[i i$, $j j]$. Similarly, the cluster centers can be expressed as

$$
v_{i k}[i i]=\frac{A_{i k}[i i]+C_{i k}[i i]}{B_{i}[i i]+D_{i}[i i]},
$$

where

$$
\begin{aligned}
A_{i k}[i i] & =\sum_{j=1}^{n} u_{i j}^{2}[i i] x_{j k}, \\
B_{i}[i i] & =\sum_{j=1}^{n} u_{i j}^{2}[i i], \\
C_{i k}[i i] & =\sum_{\substack{j j=1 \\
j \neq \neq i i}}^{p} \alpha[i i, j j] \sum_{j=s}^{n}\left(u_{i j}[i i]-u_{i j}[j j]\right)^{2} x_{j k}, \\
D_{i}[i i] & =\sum_{\substack{j j=1 \\
j j \neq i i}}^{p} \alpha[i i, j j] \sum_{j=s}^{n}\left(u_{i j}[i i]-u_{i j}[j j]\right)^{2} .
\end{aligned}
$$

In fact, the process of collaborative clustering can be partitioned into two phases: the first stage is to execute fuzzy $c$-means clustering algorithm for each data set $X[i i]$ separately, in which case we can obtain partition matrix $U[i i]$ and cluster centers $V[i i]$. The second stage is to compute the collaborated partition matrix by (2) and the cluster centers by (3).

\section{Cluster Center-Based Ranking Approach for Complex Objects}

Here, we pay our attention to the construction of ranking algorithm when the collaborative information of each complex object is provided in terms of cluster centers. For all ranking objects $G^{(1)}, G^{(2)}, \ldots, G^{(p)}$, we suppose that they have the same number of patterns, as well as the referential object G.

In the approach to be given in this section, there are three kinds of collaborative information, producing corresponding cluster center matrices: (1) if it is derived from the attribute information of complex object $G^{(i i)}$, then we apply $\dot{V}[i i]$ to denote the collaborative information; (2) if it is derived from the relational information of complex object $G^{(i i)}$, then we apply $\ddot{V}[i i]$ to denote the collaborative information; (3) if it is derived from both of the attribute information and relational information of the complex object $G^{(i i)}$, then we apply $\breve{V}[i i]$ to denote the collaborative information. It should be noted that if the collaborative information is composed of two irrelative parts (one is derived from attribute information and another is derived from relational information), then $\{\dot{V}[i i], \ddot{V}[i i]\}$ comes naturally. Certainly, the information contained in these matrices can be combined to be embedded in collaborative fuzzy $c$-means clustering.

With the above description, one knows that the collaborative information has at least three possible representation types, and different type represents different meaning. No matter which types the collaborative information belongs to, once the collaborative mechanism is introduced to rank the objects $G^{(i i)}$ for $i i=1,2, \ldots, p$, the objective function of $G$ collaborated by $G^{(i i)}$ can be constructed as follows:

$$
Q\left(G, G^{(i i)}\right)=\sum_{i=1}^{c} \sum_{k=1}^{n} u_{i k}^{2} d^{2}\left(x_{k}, v_{i}\right)+\beta[i i] \sum_{i=1}^{c} T[i i, i],
$$

where $d\left(x_{k}, v_{i}\right)=\left\|x_{k}-v_{i}\right\|$ is the distance between pattern $x_{k}$ and center $v_{i}$ and $\beta[i i]=(\beta[i i, 1], \beta[i i, 2], \beta[i i, 3])$ is a label function with respect to the collaborative information. Moreover, $T[i i, i]$ is the distance between $v_{i} \in V$ and the collaborative information $V[i i]$ :

$$
T[i i, i]=\left(\begin{array}{l}
\sum_{j=1}^{c_{1}}\left\|v_{i}-\dot{v}_{j}[i i]\right\|^{2} \\
\sum_{j=1}^{c_{2}}\left\|v_{i}-\ddot{v}_{j}[i i]\right\|^{2} \\
\sum_{j=1}^{c_{3}}\left\|v_{i}-\breve{v}_{j}[i i]\right\|^{2}
\end{array}\right) .
$$

Obviously, in (5) $\beta[i i] \sum_{i=1}^{c} T[i i, i]$ describes the collaboration influence of $G^{(i i)}$ on $G$, and parameter $\beta[i i]$ can be viewed as the collaborative coefficient that is relevant to the types of collaborative information. Meanwhile, (6) represents the distances between the cluster center $v_{i}$ of referential object $G$ and the cluster center $v_{i}[i i]$ of complex object $G^{(i i)}$.

Remark 1. The collaborative coefficient $\beta[i i]$ can be determined from the types of the collaborative information:

(i) If the collaborative information is $\dot{V}[i i]$, then $\beta[i i$, $2]=\beta[i i, 3]=0$ and $\beta[i i, 1] \neq 0$.

(ii) If the collaborative information is $\ddot{V}[i i]$, then $\beta[i i$, $1]=\beta[i i, 3]=0$ and $\beta[i i, 2] \neq 0$.

(iii) If the collaborative information is $\breve{V}[i i]$, then $\beta[i i$, $1]=\beta[i i, 2]=0$ and $\beta[i i, 3] \neq 0$.

(iv) If the collaborative information is $\{\dot{V}[i i], \ddot{V}[i i]\}$, then $\beta[i i, 3]=0$, but $\beta[i i, 1] \neq 0$ and $\beta[i i, 2] \neq 0$.

As can be seen from Remark 1, the types of collaborative information can determine which component of $\beta[i i]$ is equal to 0 , but the value of other nonzero components is not provided. No matter which types of collaborative information, that is, the collaborative information is $\dot{V}[i i], \ddot{V}[i i], \breve{V}[i i]$, and/or $\{\dot{V}[i i], \ddot{V}[i i]\}$, the concrete value of $\beta[i i]$ depends entirely on the requirement of practical ranking problem, and it can be calculated by many methods.

So far, one can regard the objective function in (5) as an optimization problem with the constraint $\sum_{i=1}^{c} u_{i k}=1$. In what follows, we still apply the Lagrange multiplier method to solve it. At first, the Lagrange function of (5) can be rewritten as

$$
\begin{aligned}
L_{\lambda}\left(G, G^{(i i)}\right)= & \sum_{i=1}^{c} u_{i k}^{2} d^{2}\left(x_{k}, v_{i}\right)+\beta[i i] \sum_{i=1}^{c} T[i i, i] \\
& -\lambda\left(\sum_{i=1}^{c} u_{i k}-1\right)
\end{aligned}
$$


Computing the derivative of $L_{\lambda}\left(G, G^{(i i)}\right)$ with respect to $u_{i k}$ and making it equal to 0 , we have

$$
\begin{aligned}
\frac{\partial L_{\lambda}\left(G, G^{(i i)}\right)}{\partial u_{i k}} & =2 u_{i k} d^{2}\left(x_{k}, v_{i}\right)-\lambda=0, \\
u_{i k} & =\frac{\lambda}{2 d^{2}\left(x_{k}, v_{i}\right)} .
\end{aligned}
$$

On account of the constraint $\sum_{i=1}^{c} u_{i k}=1$, we have that the Lagrange multiplier $\lambda$ can be determined as

$$
\lambda=\frac{1}{\sum_{i=1}^{c}\left(2 d^{2}\left(x_{k}, v_{i}\right)\right)^{-1}} .
$$

Inserting the expression of the above into (9) yields

$$
u_{i k}=\left(\sum_{j=1}^{c}\left(\frac{\left\|x_{k}-v_{i}\right\|}{\left\|x_{k}-v_{j}\right\|}\right)^{2}\right)^{-1} .
$$

The computations of the cluster centers are straightforward, as no constraints are imposed on them. By executing the derivation of (5) with respect to variant $v_{i}$, and letting the result be equal to 0 , then we have that

$$
\frac{\partial Q}{\partial v_{i}}=-2 \sum_{k=1}^{n} u_{i k}^{2}\left(x_{k}-v_{i}\right)+\beta[i i] \frac{\partial T[i i, i]}{\partial v_{i}}=0 .
$$

For the solving of $\partial T[i i, i] / \partial v_{i}$, by (6), we have that

$$
\frac{\partial T[i i, i]}{\partial v_{i}}=2\left(\begin{array}{c}
\sum_{j=1}^{c_{1}}\left(v_{i}-\dot{v}_{j}[i i]\right) \\
\sum_{j=1}^{c_{2}}\left(v_{i}-\ddot{v}_{j}[i i]\right) \\
\sum_{j=1}^{c_{3}}\left(v_{i}-\breve{v}_{j}[i i]\right)
\end{array}\right)
$$

Therefore, taking (10) into (9) yields

$$
v_{i}=\frac{\sum_{k=1}^{n} u_{i k}^{2} x_{k}+\phi_{2}(i i)}{\sum_{k=1}^{n} u_{i k}^{2}+\phi_{1}(i i)}
$$

where $\phi_{1}(i i)=\beta[i i, 1] c_{1}+\beta[i i, 2] c_{2}+\beta[i i, 3] c_{3}$ and $\phi_{2}(i i)=$ $\beta[i i, 1] \sum_{j=1}^{c_{1}} \dot{v}_{j}[i i]+\beta[i i, 2] \sum_{j=1}^{c_{2}} \ddot{v}_{j}[i i]+\beta[i i, 3] \sum_{j=1}^{\mathcal{C}_{3}} \breve{v}_{j}[i i]$.

Up to now, the partition matrix $U[i i]$ in (11) and the cluster centers $V[i i]$ in (14) can be used to describe the clustering results of referential object $G$ under the collaboration of complex object $G^{(i i)}$. Notice that, throughout this section, the notation " $V[i i]$ " denotes the cluster centers or collaborative information of the ranking object $G^{(i i)}$. To avoid unnecessary ambiguity, in the rest of this subsection, we apply $V_{G}[i i]$ to replace the computing results of (14).

By the collaboration of $G^{(i i)}$, one can obtain the new partition matrix $U[i i]$ and cluster centers $V_{G}$ [ii] of the referential data $G$. Certainty, if the difference of $U[i i]$ and $U$ is smaller, then we can say that $G$ and $G^{(i i)}$ have a bigger similarity in aspects of partition matrix. Similarly, if the difference of $V$ [ii] and $V_{G}[i i]$ is smaller, then we can say that $G$ and $G^{(i i)}$ have a bigger similarity in aspect of cluster center.

Bearing this in mind, the equation

$$
S_{V}\left(V, V_{G}[i i]\right)=\frac{\sum_{v \in V} \sum_{v_{i} \in V_{G}[i]} s\left(v, v_{i}\right)}{|V|\left|V_{G}[i i]\right|}
$$

can be used to compute the similarity of $V_{G}[i i]$ and $V$ about the referential object $G$, where $s\left(v, v_{i}\right)$ represents the similarity of $v \in V$ and $v_{i} \in V_{G}$ [ii], and can be calculated by many methods [38]. Similarly, the equation

$$
S_{U}(U, U[i i])=\frac{\sum_{i=1}^{c} \sum_{k=1}^{n} u_{i k} u_{i k}[i i]}{\|U\|\|U[i i]\|}
$$

can be used to calculate the similarity of $U$ and $U[i i]$, where $\|U[i i]\|=\sqrt{\sum_{i=1}^{c} \sum_{k=1}^{n} u_{i k}^{2}[i i]}$. By (15) and (16), the similarity of clustering results of referential object $G$ before and after collaboration of $G^{(i i)}$ can be determined by the following equation:

$$
S\left(G, G^{(i i)}\right)=\frac{S_{U}^{2}(U, U[i i])+S_{V}^{2}\left(V, V_{G}[i i]\right)}{S_{U}(U, U[i i])+S_{V}\left(V, V_{G}[i i]\right)}
$$

Obviously, if the similarity between $G$ and $G^{(i i)}$ is greater than that of $G$ and $G^{(j j)}$, then $G^{(i i)}$ is nearer to $G$ than the object $G^{(j j)}$ in aspects of collaborative information. Therefore, complex object $G^{(i i)}$ should be sorted in front of complex object $G^{(j j)}$. So much for this, the collaborative clusteringbased ranking approach for complex objects can be summarized in Algorithm 1.

\section{Partition Matrix-Based Ranking Approach for Complex Objects}

In the partition matrix-based ranking approach for complex objects, the collaborative information provided by each ranking object is the corresponding partition matrix. Similarly, we suppose that the referential object $G$ and the ranking object $G^{(i i)}$ for $i i=1,2, \ldots, p$ have the same number of patterns.

Similar to what was discussed in Section 3, there are three kinds of collaborative information, producing corresponding partition matrices: (1) if it is derived from the attribute information of $G^{(i i)}$, then we apply $\dot{U}[i i]=\left(\dot{u}_{i k}[i i]\right)_{c_{1} \times n}$ to denote the collaborative information; (2) if it is derived from the relational information of $G^{(i i)}$, then we apply $\ddot{U}[i i]=\left(\ddot{u}_{i k}[i i]\right)_{\mathcal{C}_{2} \times n}$ to denote the collaborative information; (3) and if it is derived from both of the attribute information and relational information of $G^{(i i)}$, then we apply $\breve{U}[i i]=\left(\breve{u}_{i k}[i i]\right)_{\mathcal{c}_{3} \times n}$ to denote the collaborative information. Certainly, if the collaborative information is composed of two irrelative parts (one is derived from attribute information and another is derived from relational information), then $\{\dot{U}[i i], \ddot{U}[i i]\}$ comes naturally.

In terms of the cluster center numbers $c, c_{1}, c_{2}$, and $c_{3}$, one has $c=c_{i}$ and $c \neq c_{i}$. Because of the complexity of 
Input: referential object $G$; collaborative information $V[i i], \beta[i i]$ for $i i=1,2, \ldots, p ; \varepsilon, \eta$.

Output: possible sequence $G^{(i 1)} \geqslant G^{(i 2)} \geqslant \cdots \geqslant G^{(i q)}$.

begin

(1) Compute the partition matrix $U$ and cluster center $V$ of $G$ by some clustering algorithm.

(2) For each object $G^{(i i)}$, compute the partition matrix $U[i i]$ by (11) and the cluster center $V_{G}[i i]$ by (14).

(3) For each object $G^{(i i)}$, compute the similarity of clustering results about the referential object $G$ before and after collaboration of complex object $G^{(i)}$ by (17).

(4) Compare the results computed by (17): if $S\left(G, G^{(i i)}\right) \geqslant S\left(G, G^{(j j)}\right)$, then $G^{(i i)} \geqslant G^{(j j)}$, otherwise $G^{(j j)}>G^{(i i)}$. end

Algorithm 1: Cluster center-based ranking approach for complex objects.

the representation of collaborative information $\{\dot{U}[i i], \ddot{U}[i i]\}$, at first we make a discussion on $\dot{U}[i i], \ddot{U}[i i]$, and $\breve{U}[i i]$, respectively. By the description of collaborative clustering, if $c=c_{i}$, then the objective function can be constructed as follows:

$$
\begin{aligned}
& Q\left(G, G^{(i i)}\right) \\
& =\sum_{i=1}^{c} \sum_{k=1}^{n} u_{i k}^{2} d^{2}\left(x_{k}, v_{i}\right) \\
& \quad+\delta[i i] \sum_{i=1}^{c} \sum_{k=1}^{n}\left(u_{i k}-u_{i k}[i i]\right)^{2} d^{2}\left(x_{k}, v_{i}\right),
\end{aligned}
$$

where $\delta[i i]$ is the decision maker's confidence for the collaborative information.

Obviously, in (18) the part $\delta[i i] \sum_{i=1}^{c} \sum_{k=1}^{n}\left(u_{i k}-\right.$ $\left.u_{i k}[i i]\right)^{2} d^{2}\left(x_{k}, v_{i}\right)$ is the collaboration of $G^{(i i)}$ to $G$, and $\delta[i i]$ can be viewed as the collaborative coefficient. Note that the mechanism of parameter $\delta[i i]$ is the same as that of parameter $\beta[i i]$.

If $c \neq c_{i}$, the objective function in (18) will be invalid. By taking the fact that each row of the partition matrix represents the classification distribution as well as the concept of proximity proposed in $[39,40]$ into consideration, here (18) can be rewritten as follows:

$$
\begin{aligned}
& Q\left(G, G^{(i i)}\right) \\
& =\sum_{i=1}^{c} \sum_{k=1}^{n} u_{i k}^{2} d^{2}\left(x_{k}, v_{i}\right) \\
& \quad+\delta[i i] \sum_{k=1}^{n} \sum_{s=1}^{n}\left(p_{i k}-p_{i k}[i i]\right)^{2} d^{2}\left(x_{k}, x_{s}\right),
\end{aligned}
$$

where $p_{k s}$ and $p_{k s}[i i]$ can be computed by formula $p_{i j}=$ $\sum_{k=1}^{c}\left(u_{k i} \wedge u_{k j}\right)$. Up to now, under the constraint $\sum_{i=1}^{c} u_{i k}=$ 1 , the objective function in (19) can be solved by Lagrange multiplier method.

Next, we discuss the problem of how to construct the objective function when the collaborative information is
$\{\dot{U}[i i], \ddot{U}[i i]\}$. By taking $c=c_{i}$ and $c \neq c_{i}$ into consideration, the objective function can be constructed as

$$
\begin{aligned}
Q\left(G, G^{(i i)}\right)= & \sum_{i=1}^{c} \sum_{k=1}^{n} u_{i k}^{2} d^{2}\left(x_{k}, v_{i}\right) \\
& +\delta[i i] \sum_{k=1}^{n} \sum_{s=1}^{n} \Delta_{i k}[i i] d^{2}\left(x_{k}, v_{s}\right) \\
& +\gamma[i i] \sum_{k=1}^{n} \sum_{s=1}^{n} \nabla_{i k}[i i] d^{2}\left(x_{k}, v_{s}\right),
\end{aligned}
$$

where $\delta[i i]=(\delta[i i, 1], \delta[i i, 2], \delta[i i, 3])$ is a label function with respect to $c=c_{i}$ and $\gamma[i i]=(\gamma[i i, 1], \gamma[i i, 2], \gamma[i i, 3])$ is also a label function with respect to $c \neq c_{i}$. Moreover, $\Delta_{k s}[i i]$ can be defined as

$$
\Delta_{k s}[i i]=\left(\begin{array}{c}
\left(u_{i k}-\dot{u}_{i k}[i i]\right)^{2} \\
\left(u_{i k}-\ddot{u}_{i k}[i i]\right)^{2} \\
\left(u_{i k}-\breve{u}_{i k}[i i]\right)^{2}
\end{array}\right)
$$

and $\nabla_{k s}[i i]$ that represents the distance between objects $x_{k}$ and $x_{s}$ can be defined as

$$
\nabla_{k s}[i i]=\left(\begin{array}{c}
\left(p_{k s}-\dot{u}_{k s}[i i]\right)^{2} \\
\left(p_{k s}-\ddot{p}_{k s}[i i]\right)^{2} \\
\left(p_{k s}-\breve{p}_{k s}[i i]\right)^{2}
\end{array}\right),
$$

where $\dot{p}_{k s}[i i], \ddot{p}_{k s}[i i]$, and $\breve{p}_{k s}[i i]$ can be determined by formula $p_{i j}=\sum_{k=1}^{c}\left(u_{k i} \wedge u_{k j}\right)$.

Remark 2. Similarly, (18)-(22) describe the collaboration of complex object $G^{(i i)}$ to the referential object $G$. The collaborative coefficient $\delta[i i]$ and $\gamma[i i]$ can be determined from the types of the collaborative information:

(i) The collaborative information is $\dot{U}[i i]$ : if $c=c_{1}$, then there is only $\delta[i i, 1] \neq 0$; otherwise there is only $\gamma[i i, 1] \neq 0$.

(ii) The collaborative information is $\ddot{U}[i i]$ : if $c=c_{2}$, then there is only $\delta[i i, 2] \neq 0$; otherwise there is only $\gamma[i i, 2] \neq 0$. 
(iii) The collaborative information is $\breve{U}[i i]$ : if $c=c_{3}$, then there is only $\delta[i i, 3] \neq 0$; otherwise there is only $\gamma[i i, 3] \neq 0$.

(iv) The collaborative information is $\{\dot{U}[i i], \ddot{U}[i i]\}$ : if $c=$ $c_{1}$ and $c=c_{2}$, then there are only $\delta[i i, 1] \neq 0$ and $\delta[i i, 2] \neq 0$; if $c_{1} \neq c$ and $c_{2} \neq c$, then there are only $\gamma[i i, 1] \neq 0$ and $\gamma[i i, 2] \neq 0$; if $c_{1}=c$ and $c_{2} \neq c$, then there are only $\delta[i i, 1] \neq 0$ and $\gamma[i i, 2] \neq 0$; if $c_{1} \neq c$ and $c_{2}=c$, then there are only $\gamma[i i, 1] \neq 0$ and $\delta[i i, 2] \neq 0$.

Evidently, types of collaborative information have a critical implication for the value selection of parameters $\delta[i i]$ and $\gamma[i i]$. No matter which types it belongs to, the parameters can be predetermined or provided by decision makers during the process of ranking.

Just as described in foregoing sections, next we still employ the Lagrange multiplier method to solve the ranking problem based on (20). Let the Lagrange function of (20) be

$$
\begin{aligned}
L_{\lambda}\left(G, G^{(i i)}\right)= & \sum_{i=1}^{c} u_{i k}^{2} d^{2}\left(x_{k}, v_{i}\right) \\
& +\delta[i i] \sum_{i=1}^{c} \Delta_{i k}[i i] d^{2}\left(x_{k}, v_{i}\right) \\
& +\gamma[i i] \sum_{i=1}^{c} \nabla_{i k}[i i] d^{2}\left(x_{k}, x_{s}\right) \\
& -\lambda\left(\sum_{i=1}^{c} u_{i k}-1\right),
\end{aligned}
$$

where $\lambda$ is the Lagrange multiplier.

Computing the derivative of $L_{\lambda}\left(G, G^{(i)}\right)$ with respect to $u_{i k}$ and making it equal to 0 , we have

$$
\begin{aligned}
\frac{\partial L_{\lambda}}{\partial u_{i k}}= & \gamma[i i] \sum_{s=1}^{n} \frac{\partial \nabla_{k s}[i i]}{\partial u_{i k}} d^{2}\left(x_{k}, x_{s}\right) \\
& +\delta[i i] \frac{\partial \Delta_{k s}[i i]}{\partial u_{i k}} d^{2}\left(x_{k}, v_{i}\right)+2 u_{i k} d^{2}\left(x_{k}, v_{i}\right) \\
& -\lambda=0 .
\end{aligned}
$$

In addition, taking (21) into variant $\Delta_{i k}[i i]$, we have

$$
\frac{\partial \Delta_{p s}[i i]}{\partial u_{i k}}=2\left(\begin{array}{c}
u_{i k}-\dot{u}_{i k}[i i] \\
u_{i k}-\ddot{u}_{i k}[i i] \\
u_{i k}-\breve{u}_{i k}[i i]
\end{array}\right)
$$

Similarly, by computing, we have

$$
\frac{\partial \nabla_{p s}[i i]}{\partial u_{i k}}=2\left(\begin{array}{c}
p_{k s}-\dot{p}_{k s}[i i] \\
p_{k s}-\ddot{p}_{k s}[i i] \\
p_{k s}-\breve{p}_{k s}[i i]
\end{array}\right) \frac{\partial p_{k s}[i i]}{\partial u_{i k}}
$$

where $\partial p_{k s}[i i] / \partial u_{i k}=1$ if $s \in \Gamma$; otherwise $\partial p_{k s}[i i] / \partial u_{i k}=0$. Note that $\Gamma$ is an index set and can be described as $k_{0} \in \Gamma$ if and only if there exists one $k_{0} \in\{1,2, \ldots, n\}$ such that $u_{i k} \leq$ $u_{i k_{0}}$ and $\bigvee_{i \neq j}^{c}\left(u_{j k} \wedge u_{j k_{0}}\right) \leqslant u_{i k} \wedge u_{i k_{0}}$. Taking (26) and (25) into (24), we have that

$$
\begin{aligned}
\lambda= & 2 u_{i k}\left\|x_{k}-v_{i}\right\|^{2}+2 d^{2}\left(x_{k}, v_{i}\right) \sum_{j=1}^{3} \delta[i i, j] u_{i k} \\
& +\phi(i i, k)-2 \delta[i i] d^{2}\left(x_{k}, v_{i}\right) U[i i, i, k],
\end{aligned}
$$

where $U[i i, i, k]=\left(\dot{u}_{i k}[i i], \ddot{u}_{i k}[i i], \breve{u}_{i k}[i i]\right)^{T}$ and

$$
\phi(i i, k)=2 \gamma[i i] \sum_{s \in T}\left(\begin{array}{c}
p_{k s}-\dot{p}_{k s}[i i] \\
p_{k s}-\ddot{p}_{k s}[i i] \\
p_{k s}-\breve{p}_{k s}[i i]
\end{array}\right) d^{2}\left(x_{k}, x_{s}\right) .
$$

Obviously, (27) can be rewritten as

$$
u_{i k}=\frac{\lambda-\varphi_{1}(i i, i, k)}{2\left\|x_{k}-v_{i}\right\|^{2} \varphi_{2}[i i]}
$$

where $\varphi_{1}(i i, i, k)=\phi(i i, k)-2 \delta[i i]\left\|x_{k}-v_{i}\right\|^{2} U[i i, i, k]$ and $\varphi_{2}[i i]=1+\sum_{t=1}^{3} \delta[i i, t]$. By taking the above equation into constraint $\sum_{i=1}^{c} u_{i k}=1$, we have that

$$
\lambda=\frac{1+\sum_{i=1}^{c}\left(\varphi_{1}(i i, i, k) / 2 d^{2}\left(x_{k}, v_{i}\right) \varphi_{2}[i i]\right)}{\sum_{i=1}^{c}\left(1 / 2 d^{2}\left(x_{k}, v_{i}\right) \varphi_{2}[i i]\right)} .
$$

Taking (30) into (27), we have

$$
\begin{aligned}
& u_{i k} \\
& =\frac{1+\sum_{j=1}^{c}\left(\left(\varphi_{1}(i i, j, k)-\varphi_{1}(i i, i, k)\right) / 2 d^{2}\left(x_{k}, v_{i}\right) \varphi_{2}[i i]\right)}{\sum_{i=1}^{c}\left(d^{2}\left(x_{k}, v_{i}\right) / d^{2}\left(x_{k}, v_{j}\right)\right)} .
\end{aligned}
$$

The computation of the cluster centers is straightforward, as no constraints are imposed on them. By executing the derivation of (20) with respect to variant $v_{i}$, and letting the result be equal to 0 , then we have that

$$
\sum_{k=1}^{n} u_{i k}^{2}\left(x_{k}-v_{i}\right)+\delta[i i] \sum_{k=1}^{n} \Delta_{i k}[i i]\left(x_{k}-v_{i}\right)=0,
$$

which yields

$$
v_{i}=\frac{\sum_{k=1}^{n} u_{i k}^{2} x_{k}+\delta[i i] \sum_{k=1}^{n} \Delta_{i k}[i i] x_{k}}{\sum_{k=1}^{n} u_{i k}^{2}+\delta[i i] \sum_{k=1}^{n} \Delta_{i k}[i i]} .
$$

Up to now, the partition matrix determined by (31) and the cluster centers determined by (33) can be applied to describe the clustering results of the referential object $G$ under the collaboration of complex object $G^{(i i)}$. Notice that, throughout this section, the notation " $U[i i]$ " denotes the partition matrix or the collaborative information of the complex object $G^{(i i)}$. To avoid unnecessary ambiguity, in the rest of this subsection, we apply $U_{G}[i i]$ to describe the computing results of (31). 
Input: referential object $G$; collaborative information $U[i i], \delta[i i]$ and $\gamma[i i]$ for $i i=1,2, \ldots, p ; \varepsilon, \eta$.

Output: possible sequence $G^{(i 1)} \geqslant G^{(i 2)} \geqslant \cdots \geqslant G^{(i q)}$.

begin

(1) Compute the partition matrix $U$ and cluster center $V$ of $G$ by some clustering algorithm.

(2) For each object $G^{(i i)}$, compute the partition matrix $U_{G}[i i]$ by (31) and the cluster center $V$ [ii] by (33).

(3) For each object $G^{(i i)}$, compute the similarity of clustering results about $G$ before and after collaboration of object $G^{(i i)}$ by (34).

(4) Compare the results computed by (34): if $S\left(G, G^{(i i)}\right) \geqslant S\left(G, G^{(j j)}\right)$, then $G^{(i i)} \geqslant G^{(j j)}$, otherwise $G^{(j j)}>G^{(i i)}$. end

Algorithm 2: Partition matrix-based ranking approach for complex objects.

Similarly, the similarity between $G$ and $G^{(i i)}$ can be determined by equation

$$
S\left(G, G^{(i)}\right)=\frac{S_{V}^{2}(V, V[i i])+S_{U}^{2}\left(U, U_{G}[i i]\right)}{S_{V}(V, V[i i])+S_{U}\left(U, U_{G}[i i]\right)} .
$$

So much for this, the collaborative clustering-based ranking approach for complex objects can be summarized in Algorithm 2.

\section{Experimental Studies}

In this section, we make an experimental analysis about the proposed ranking approaches for complex objects by simulating some data sets.

\subsection{Example of Cluster Center-Based Ranking Approach.} Given that $G^{(i i)}$ for $i i=1,2, \ldots, 5$ are ranking objects, and $G$ is the referential object, Figures 1 and 2 show the attribute information and the partition matrix of $G$, respectively. The cluster centers $V$ of the referential object $G$ are $v_{1}=(6.5,2.9)$, $v_{2}=(5.5,2.8)$, and $v_{3}=(5.3,2.4)$. The collaborative information of complex objects $\breve{V}[i i]$ for $i i=1,2, \ldots, 5$ is listed in Table 1.

Since the cluster number of referential object $G$ is $c=3$ and the collaborative information is $\breve{V}[i i], \beta[i i, 1]=\beta[i i, 2]=$ 0 holds. For the determination of $\beta[i i, 3]$, here we use the following formula:

$$
\beta[i i, 3]= \begin{cases}1 & \text { if } c=c_{3} \\ \frac{c \wedge c_{3}}{c \vee c_{3}} & \text { if } c \neq c_{3} .\end{cases}
$$

Based on this, parameter $\beta[i i]$ can be expressed as $(0,0,1)$ for $G^{(1)}, G^{(2)}$, and $G^{(4)}$ and $(0,0,2 / 3)$ for $G^{(3)}$ and $G^{(5)}$.

By computing, we can obtain the new partition matrix of referential object $G$ under the collaboration of the ranking objects (shown in Figure 3 ) and the cluster centers (listed in Table 2).

According to the description of Algorithm 1, we have

$$
\begin{aligned}
& S\left(V, V_{G}[1]\right)=0.9940, \\
& S\left(V, V_{G}[2]\right)=0.9979,
\end{aligned}
$$

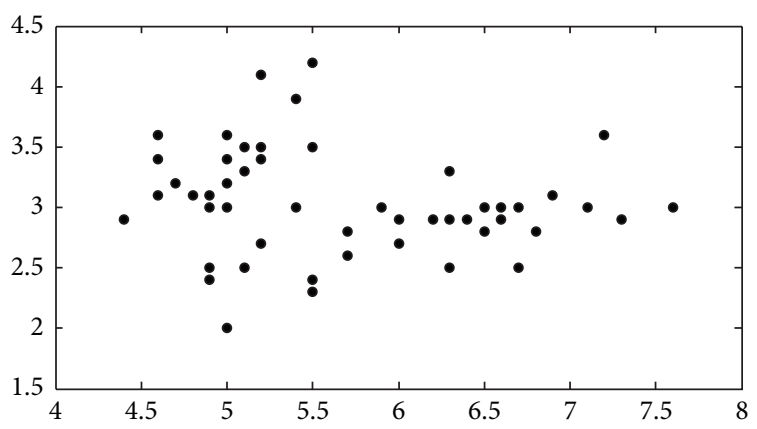

FIGURE 1: The attribute information of referential object $G$

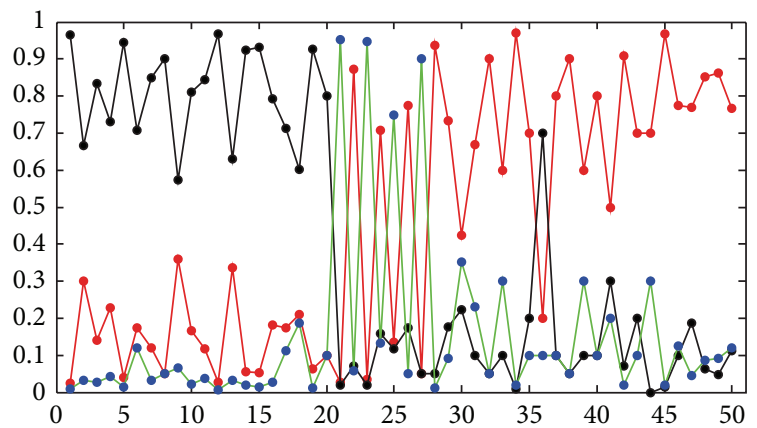

FIgURE 2: The partition matrix $U$ of referential object $G$.

TABLE 1: The collaborative information provided by complex objects.

\begin{tabular}{lllllllllll}
\hline & \multicolumn{2}{c}{$G^{(1)}$} & \multicolumn{2}{c}{$G^{(2)}$} & \multicolumn{2}{c}{$G^{(3)}$} & \multicolumn{2}{c}{$G^{(4)}$} & \multicolumn{2}{c}{$G^{(5)}$} \\
\hline$v_{1}$ & 5.3 & 2.5 & 5.0 & 3.0 & 5.0 & 3.3 & 5.3 & 3.3 & 5.0 & 3.4 \\
$v_{2}$ & 5.0 & 3.3 & 5.1 & 2.5 & 5.3 & 2.5 & 6.6 & 2.9 & 6.5 & 2.9 \\
$v_{3}$ & 6.0 & 2.0 & 6.2 & 2.8 & & & 5.3 & 2.5 & & \\
\hline
\end{tabular}

TABLE 2: The new cluster centers of the referential object $G$.

\begin{tabular}{lllllllllll}
\hline & \multicolumn{3}{c}{$G^{(1)}$} & \multicolumn{2}{c}{$G^{(2)}$} & \multicolumn{2}{c}{$G^{(3)}$} & \multicolumn{2}{c}{$G^{(4)}$} & \multicolumn{2}{c}{$G^{(5)}$} \\
\hline$v_{1}$ & 5.3 & 2.5 & 5.0 & 3.0 & 5.0 & 3.3 & 5.3 & 3.3 & 5.0 & 3.4 \\
$v_{2}$ & 5.0 & 3.3 & 5.1 & 2.5 & 5.3 & 2.5 & 6.6 & 2.9 & 6.5 & 2.9 \\
$v_{3}$ & 6.0 & 2.0 & 6.2 & 2.8 & & & 5.3 & 2.5 & & \\
\hline
\end{tabular}




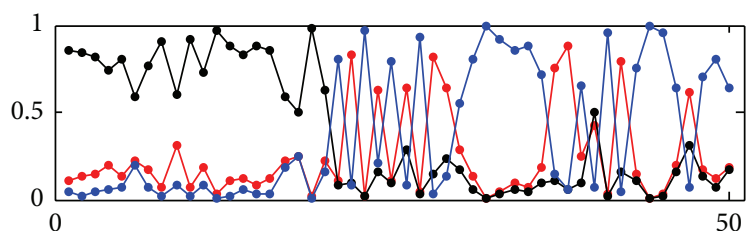

(1) $U[1]$

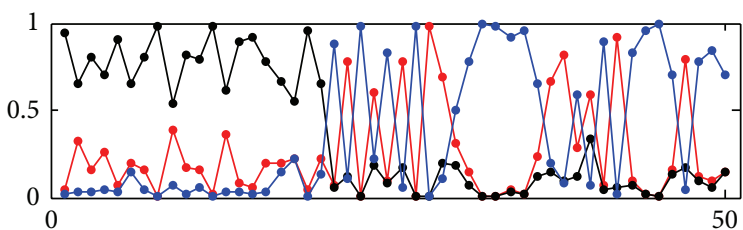

(3) $U[3]$

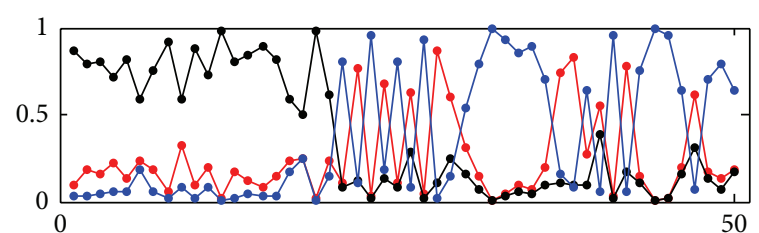

(2) $U[2]$

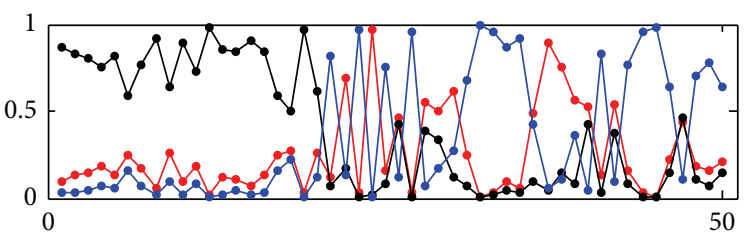

(4) $U[4]$

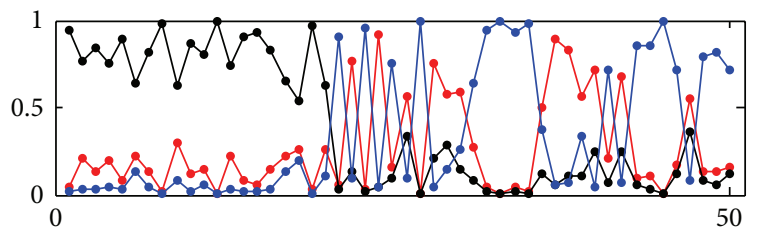

(5) $U[5]$

FIgURE 3: The new partition matrix of the referential object $G$.

$$
\begin{gathered}
S\left(V, V_{G}[5]\right)=0.9934, \\
S(U, U[1])=0.7779, \\
S(U, U[2])=0.7816, \\
S(U, U[3])=0.7798, \\
S(U, U[4])=0.7783, \\
S(U, U[5])=0.7909 .
\end{gathered}
$$

Hence, if we take the cluster centers into consideration, then we have

$$
G^{(2)}>G^{(4)}>G^{(3)}>G^{(1)}>G^{(5)}
$$

if we take the partition matrix into consideration, then we have

$$
G^{(5)}>G^{(2)}>G^{(3)}>G^{(4)}>G^{(1)}
$$

if we follow the route of Algorithm 1, then there holds

$$
G^{(5)}>G^{(2)}>G^{(4)}>G^{(3)}>G^{(1)} .
$$

5.2. Example of Partition Matrix-Based Ranking Approach. In this part, the referential object $G$ is the same as that in Section 5.1. For the ranking objects $G^{(i i)}$ for $i i=1,2, \ldots, 5$, Figure 4 gives its collaborative information, and the collaborative information for $i i=1,2, \ldots, 5$ is $\breve{U}[i i]$.

Because the cluster number of referential object $G$ is $c=3$ and the collaborative information is $\breve{U}[i i]$, then
TABLE 3: The new cluster centers of the referential object $G$.

\begin{tabular}{lllllllllll}
\hline & \multicolumn{2}{c}{$V[1]$} & \multicolumn{2}{c}{$V[2]$} & \multicolumn{2}{c}{$V[3]$} & \multicolumn{2}{c}{$V[4]$} & \multicolumn{2}{c}{$V[5]$} \\
\hline$v_{1}$ & 5.18 & 3.12 & 6.37 & 3.01 & 5.77 & 2.99 & 5.65 & 2.74 & 5.70 & 2.93 \\
$v_{2}$ & 5.75 & 3.16 & 5.11 & 3.14 & 5.08 & 3.30 & 5.09 & 3.31 & 5.33 & 3.19 \\
$v_{3}$ & 6.05 & 2.90 & 5.90 & 2.90 & 6.24 & 2.81 & 6.43 & 2.93 & 6.21 & 2.93 \\
\hline
\end{tabular}

$\delta[i i, 1]=\delta[i i, 2]=0$ and $\gamma[i i, 1]=\gamma[i i, 2]=0$. For the determination of $\delta[i i, 3]$ and $\gamma[i i, 3]$, we apply the following formulas:

$$
\begin{aligned}
& \delta[i i, 3]= \begin{cases}1 & \text { if } c=c_{3} \\
\frac{c \wedge c_{3}}{c \vee c_{3}} & \text { if } c \neq c_{3},\end{cases} \\
& \gamma[i i, 3]= \begin{cases}1 & \text { if } c=c_{3} \\
\frac{c \wedge c_{3}}{c \vee c_{3}} & \text { if } c \neq c_{3} .\end{cases}
\end{aligned}
$$

For this, the label functions are $\delta[i i]=(0,0,1)$ and $\gamma[i i]=$ $(0,0,1)$. By computing, the new partition matrix and cluster centers of the referential object $G$ are listed in Figure 5 and Table 3.

Up to now, by computing, we have

$$
\begin{aligned}
& S(V, V[1])=0.9973, \\
& S(V, V[2])=0.9976, \\
& S(V, V[3])=0.9963, \\
& S(V, V[4])=0.9965, \\
& S(V, V[5])=0.9978,
\end{aligned}
$$




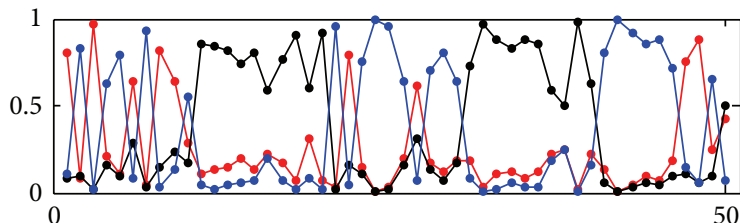

(1) $U[1]$

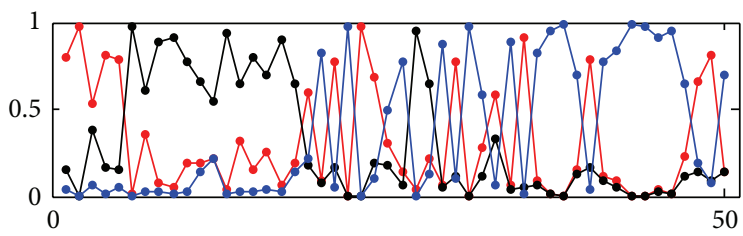

(3) $U[3]$

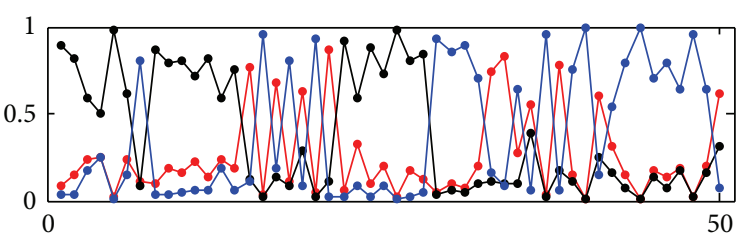

(2) $U[2]$

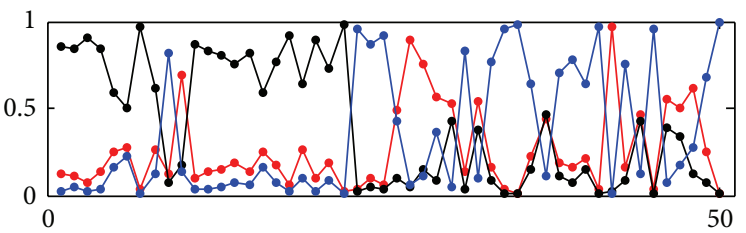

(4) $U[4]$

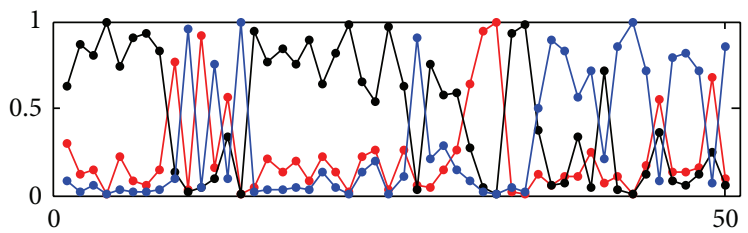

(5) $U[5]$

FIGURE 4: The provided collaborative information: partition matrix.

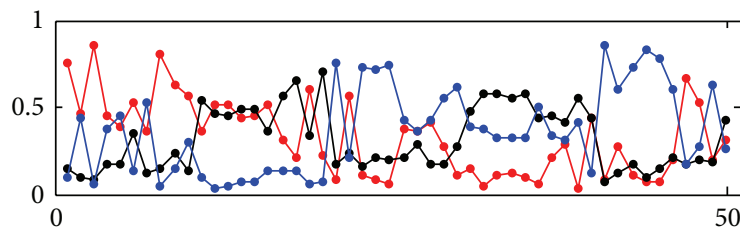

(1) $U[1]$

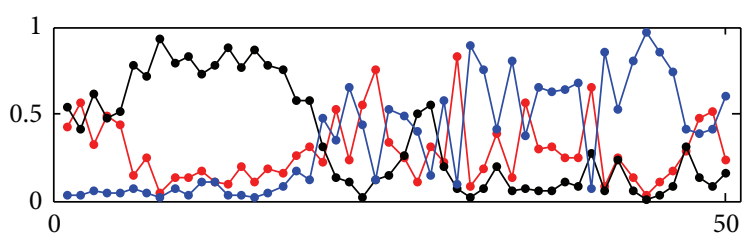

(3) $U[3]$

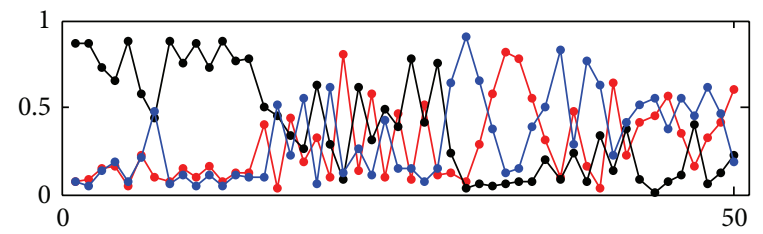

(2) $U[2]$

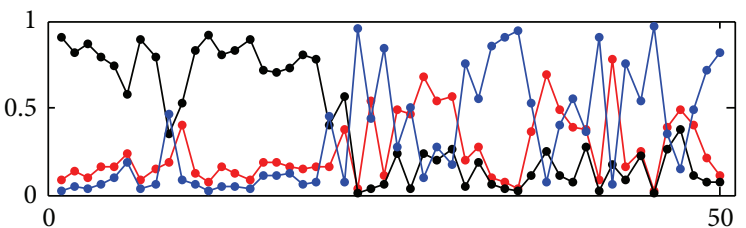

(4) $U[4]$

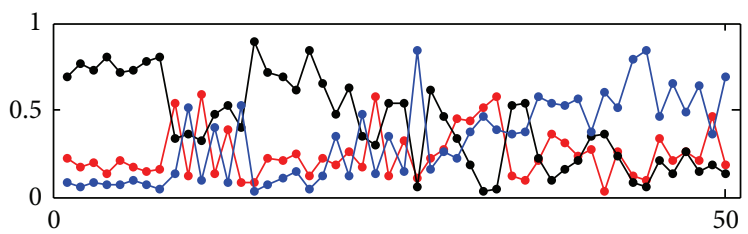

(5) $U[5]$

FIGURE 5: The new partition matrix of the referential object $G$.

$$
\begin{aligned}
& S(U, U[1])=0.5849, \\
& S(U, U[2])=0.7005, \\
& S(U, U[3])=0.7001, \\
& S(U, U[4])=0.7767, \\
& S(U, U[5])=0.7371 .
\end{aligned}
$$

Similarly, if only the partition matrix is considered, then we have

$$
G^{(4)}>G^{(5)}>G^{(2)}>G^{(3)}>G^{(1)}
$$

if only the cluster centers are considered, then we have

$$
G^{(5)}>G^{(2)}>G^{(1)}>G^{(4)}>G^{(3)}
$$


and if we apply Algorithm 2 to compute the possible sequence of the complex objects, which yields

$$
G^{(4)}>G^{(5)}>G^{(2)}>G^{(3)}>G^{(1)} .
$$

\section{Conclusions}

In this paper, the problem of ranking for complex objects is discussed carefully by considering the merits of collaborative clustering and the privacy of information. During the process of constructing the ranking algorithm, a referential object is preproposed and the collaborative information of complex objects is provided by means of partition matrices and/or cluster centers. No matter what the type of the collaborative information is, that is, the collaborative information is partition matrix or cluster center, we regarded it as an objective-function-based optimization problem and solved it by Lagrange multiplier method, where the complex objects can be sorted by comparing the difference of the referential objects before and after collaborative clustering with respect to the ranking objects. To illustrate the validity of our proposed ranking algorithms, we constructed synthetic examples and the experimental results show that our proposed algorithms are valid.

It is well known that no matter what result one ranking algorithm leads to, the topic of ranking is a soft decision making problem. We will still, in future work, pursue the analysis of the ranking approaches for complex objects. What is more, another interesting issue in this aspect is how to apply the proposed ranking methods of data with relational information into large and sparse data, in a clever, perfect way.

\section{Conflict of Interests}

The authors declare that there is no conflict of interests regarding the publication of this paper.

\section{Acknowledgments}

This work is supported by the National Natural Science Foundation of China (no. 31460297), Scientific Research Funds of Yunnan Provincial Department of Education (project name: Research on Dynamic Graph Data Clustering), Yunnan Applied Basic Research Youth Projects (project name: Research on the Sorting of Graph Data), and the Talent Introduction Research Project of Yunnan Minzu University.

\section{References}

[1] H. Sun, J. Huang, and B. Feng, "QoRank: a query-dependent ranking model using LSE-based weighted multiple hyperplanes aggregation for information retrieval," International Journal of Intelligent Systems, vol. 26, no. 1, pp. 73-97, 2011.

[2] W.-C. Hsu, C.-C. Liu, C. Fu, and S.-S. Chen, "Selecting genes for cancer classification using SVM: an adaptive multiple features scheme," International Journal of Intelligent Systems, vol. 28, no. 12, pp. 1196-1213, 2013.

[3] R. U. Bilsel, G. Büyüközkan, and D. Ruan, "A fuzzy preferenceranking model for a quality evaluation of hospital web sites," International Journal of Intelligent Systems, vol. 21, no. 11, pp. 1181-1197, 2006.
[4] G. Chechik, V. Sharma, U. Shalit, and S. Bengio, "Large scale online learning of image similarity through ranking," Journal of Machine Learning Research, vol. 11, pp. 1109-1135, 2010.

[5] A. F. Shorrocks, "Ranking income distributions," Economica, vol. 50, no. 197, pp. 3-17, 1983.

[6] G. Bortolan and R. Degani, "A review of some methods for ranking fuzzy subsets," Fuzzy Sets and Systems, vol. 15, no. 1, pp. $1-19,1985$.

[7] L. M. D. C. Ibáñez and A. G. Muñoz, "A subjective approach for ranking fuzzy numbers," Fuzzy Sets and Systems, vol. 29, no. 2, pp. 145-153, 1989.

[8] K. T. Atanassov, "Intuitionistic fuzzy sets," Fuzzy Sets and Systems, vol. 20, no. 1, pp. 87-96, 1986.

[9] Q.-S. Zhang, S. Jiang, B. Jia, and S. Luo, "Some information measures for interval-valued intuitionistic fuzzy sets," Information Sciences, vol. 180, no. 24, pp. 5130-5145, 2010.

[10] J. Figueira, S. Greco, and M. Ehrgott, Multiple Criteria Decision Analysis: State of the Art Surveys, Kluwer Academic Publishers, London, UK, 2005.

[11] S. Liu and T. A. Moughal, "A novel method for dynamic multicriteria decision making with hybrid evaluation information," Journal of Applied Mathematics, vol. 2014, Article ID 864628, 11 pages, 2014.

[12] N. Adler, L. Friedman, and Z. Sinuany-Stern, "Review of ranking methods in the data envelopment analysis context," European Journal of Operational Research, vol. 140, no. 2, pp. 249$265,2002$.

[13] G.-H. Tzeng and J.-J. Huang, Multiple Attribute Decision Making: Methods and Applications, CRC Press, Boca Raton, Fla, USA, 2011.

[14] M. Culp and G. Michailidis, "Graph-based semisupervised learning," IEEE Transactions on Pattern Analysis and Machine Intelligence, vol. 30, no. 1, pp. 174-179, 2008.

[15] S. Džeroski, Relational Data Mining, Springer, Berlin, Germany, 2010.

[16] L. Getoor and L. Mihalkova, "Learning statistical models from relational data," in Proceedings of the ACM SIGMOD International Conference on Management of Data, pp. 1195-1198, Athens, Greece, June 2011.

[17] S. Agarwal, "Ranking on graph data," in Proceedings of the 23rd International Conference on Machine Learning (ICML '06), pp. 25-32, ACM, Pittsburgh, Pa, USA, June 2006.

[18] R. Mihalcea, "Graph-based ranking algorithms for sentence extraction, applied to text summarization," in Proceedings of the Meetings of the Association for Computational Linguistics, pp. 14, 2004.

[19] S. Lee, S.-I. Song, M. Kahng, D. Lee, and S.-G. Lee, "Random walk based entity ranking on graph for multidimensional recommendation," in Proceedings of the 5th ACM Conference on Recommender Systems (RecSys '11), pp. 93-100, October 2011.

[20] S. Agarwal, "Learning to rank on graphs," Machine Learning, vol. 81, no. 3, pp. 333-357, 2010.

[21] S. Agarwal, D. Dugar, and S. Sengupta, "Ranking chemical structures for drug discovery: a new machine learning approach," Journal of Chemical Information and Modeling, vol. 50, no. 5, pp. 716-731, 2010.

[22] R. Bekkerman, M. Bilenko, and J. Langford, Scaling up Machine Learning: Parallel and Distributed Approaches, Cambridge University Press, Cambridge, UK, 2012.

[23] Y.-J. Lai, T.-Y. Liu, and C.-L. Hwang, “TOPSIS for MODM," European Journal of Operational Research, vol. 76, no. 3, pp. 486-500, 1994. 
[24] J. C. Bezdek, Pattern Recognition with Fuzzy Objective Function Algorithms, Kluwer Academic Publishers, Dordrecht, The Netherlands, 1981.

[25] J. C. Bezdek and J. O. Harris, "Fuzzy partitions and relations; an axiomatic basis for clustering," Fuzzy Sets and Systems, vol. 1, no. 2, pp. 111-127, 1978.

[26] J. C. Dunn, "A fuzzy relative of the isodata process and its use in detecting compact wellseparated clusters," Cybernetics and Systems, vol. 3, no. 3, pp. 32-57, 1973.

[27] J. Yu and M.-S. Yang, "Optimality test for generalized FCM and its application to parameter selection," IEEE Transactions on Fuzzy Systems, vol. 13, no. 1, pp. 164-176, 2005.

[28] J. Yu and M.-S. Yang, "A generalized fuzzy clustering regularization model with optimality tests and model complexity analysis," IEEE Transactions on Fuzzy Systems, vol. 15, no. 5, pp. 904-915, 2007.

[29] J. Yu, Q. Cheng, and H. Huang, "Analysis of the weighting exponent in the FCM," IEEE Transactions on Systems, Man, and Cybernetics, Part B: Cybernetics, vol. 34, no. 1, pp. 634-639, 2004.

[30] Y. H. Qian, J. Y. Liang, P. Song, and C. Y. Dang, "On dominance relations in disjunctive set-valued ordered information systems," International Journal of Information Technology \& Decision Making, vol. 9, no. 1, pp. 9-33, 2010.

[31] Y. Qian, J. Liang, and C. Dang, "Interval ordered information systems," Computers and Mathematics with Applications, vol. 56, no. 8, pp. 1994-2009, 2008.

[32] A. Ban, L. Coroianu, and P. Grzegorzewski, "Trapezoidal approximation and aggregation," Fuzzy Sets and Systems, vol. 177, no. 1, pp. 45-59, 2011.

[33] D. Dubois and H. Prade, "Gradualness, uncertainty and bipolarity: making sense of fuzzy sets," Fuzzy Sets and Systems, vol. 192, pp. 3-24, 2012.

[34] L. F. S. Coletta, V. Lucas, E. R. Hruschka, R. J. G. B. Campello, and W. Pedrycz, "Collaborative fuzzy clustering algorithms: some refinements and design guidelines," IEEE Transactions on Fuzzy Systems, vol. 20, no. 3, pp. 444-462, 2012.

[35] W. Pedrycz, "Collaborative fuzzy clustering," Pattern Recognition Letters, vol. 23, no. 14, pp. 1675-1686, 2002.

[36] W. Pedrycz, "Algorithms of fuzzy clustering with partial supervision," Pattern Recognition Letters, vol. 3, no. 1, pp. 13-20, 1985.

[37] W. Pedrycz and J. Waletzky, "Fuzzy clustering with partial supervision," IEEE Transactions on Systems, Man, and Cybernetics, Part B: Cybernetics, vol. 27, no. 5, pp. 787-795, 1997.

[38] C. Luo, Introduction to Fuzzy Sets, Beijing Normal University Press, Beijing, China, 1989.

[39] V. Loia, W. Pedrycz, and S. Senatore, "P-FCM: a proximitybased fuzzy clustering for user-centered web applications," International Journal of Approximate Reasoning, vol. 34, no. 2-3, pp. 121-144, 2003.

[40] W. Pedrycz, V. Loia, and S. Senatore, "P-FCM: a proximitybased fuzzy clustering," Fuzzy Sets and Systems, vol. 148, no. 1, pp. 21-41, 2004. 


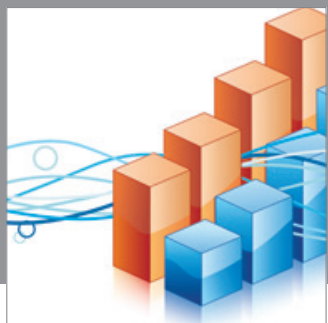

Advances in

Operations Research

mansans

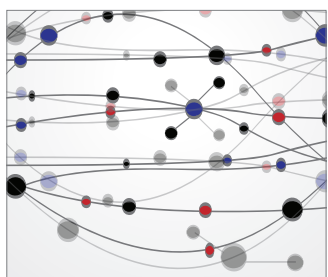

The Scientific World Journal
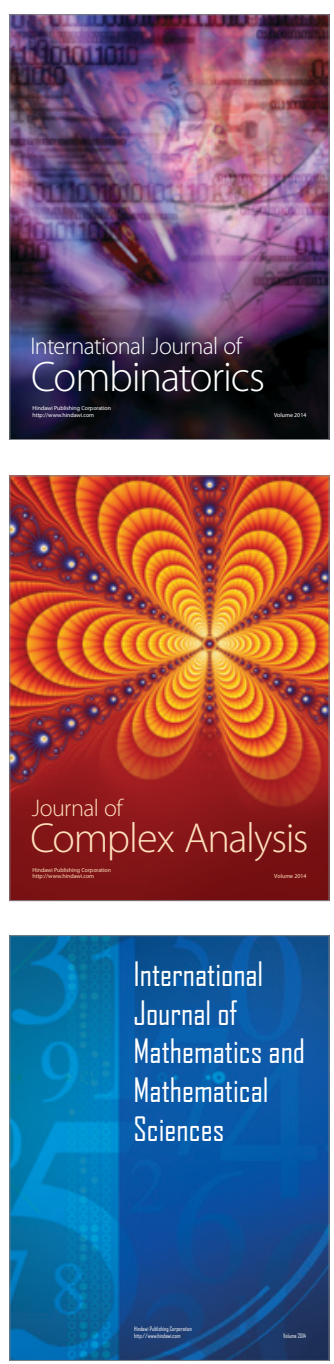
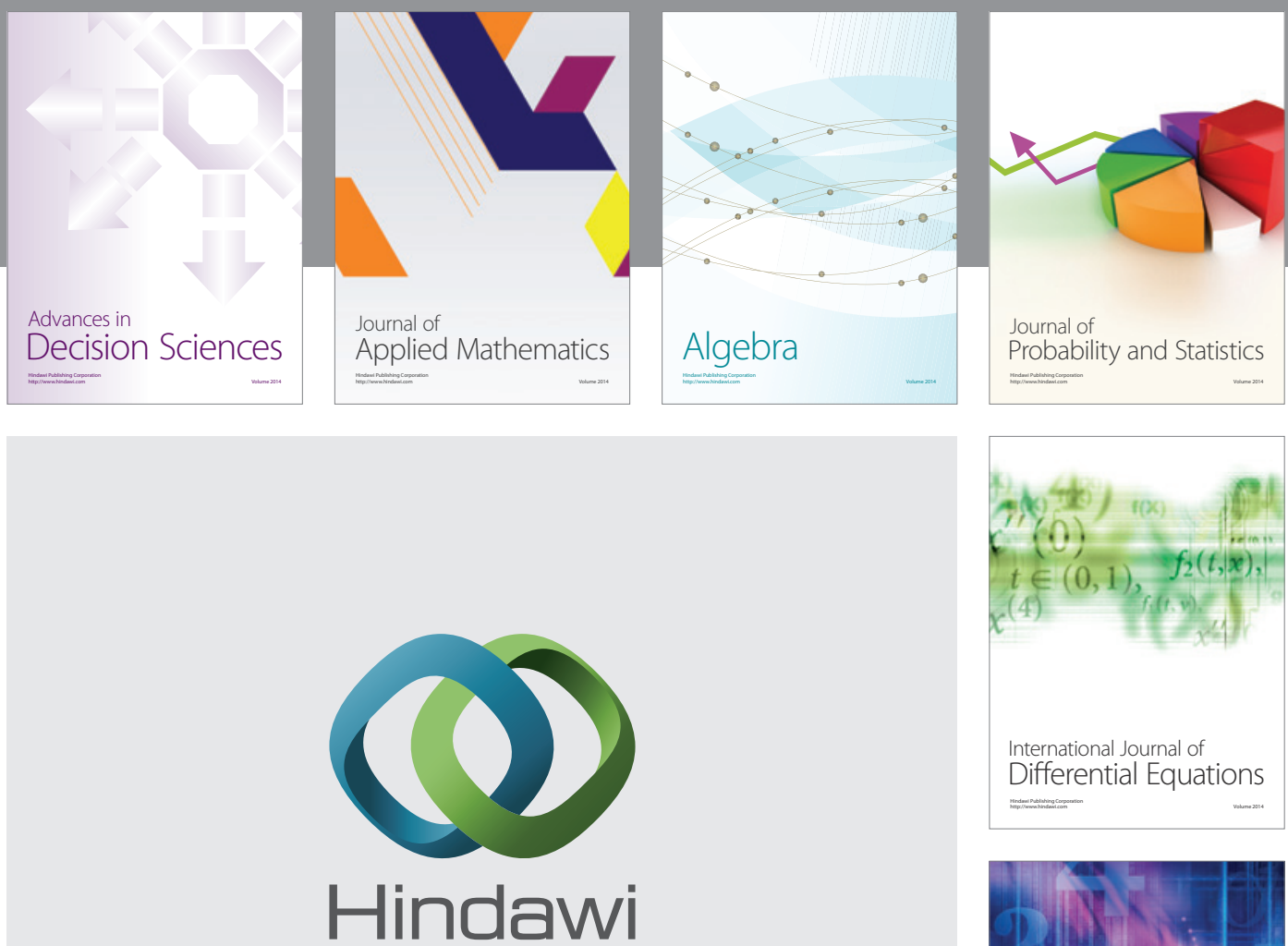

Submit your manuscripts at http://www.hindawi.com
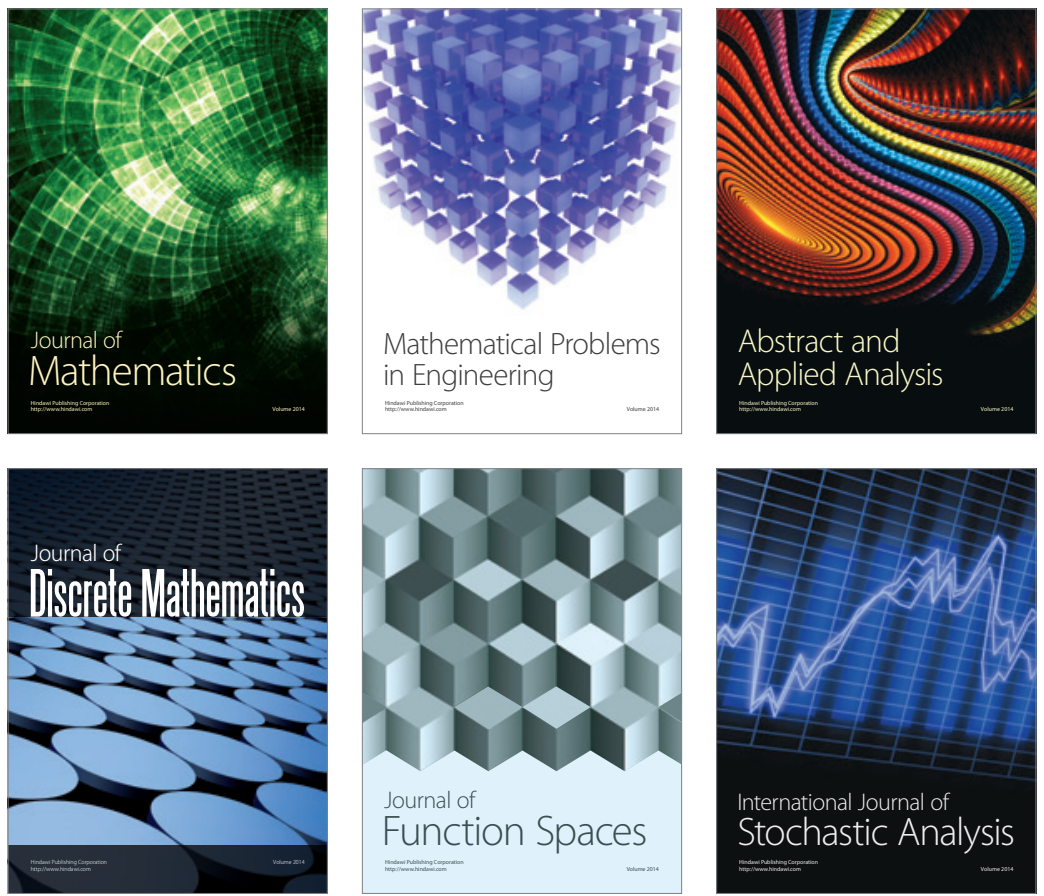

Journal of

Function Spaces

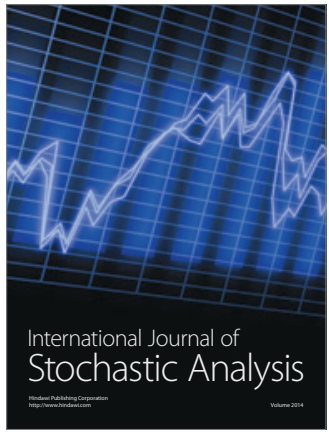

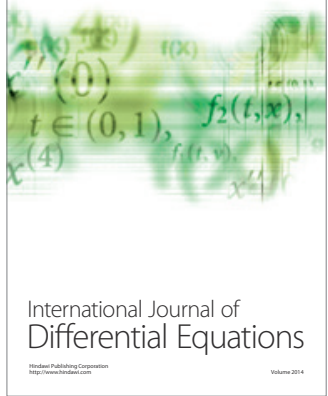
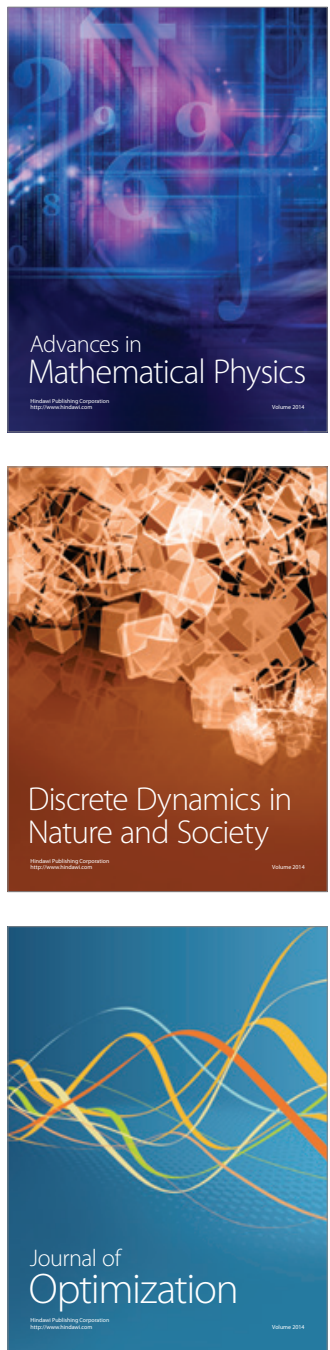